\title{
CDISC Therapeutic Area User Guide Response Terminology
}

National Cancer Institute

\section{Source}

National Cancer Institute. CDISC Therapeutic Area User Guide Response Terminology. NCl Thesaurus. Code C160925.

Terminology associated with the CDISC therapeutic area user guide response codelist of the Clinical Data Interchange Standards Consortium (CDISC) Study Data Tabulation Model (SDT M). 